\title{
Abundances and Kinematics for a Sample of LMC Clusters
}

\author{
Doug Geisler ${ }^{1}$, Aaron J. Grocholski ${ }^{2}$, Ata Sarajedini ${ }^{2}$, \\ Andrew A. Cole ${ }^{3}$, and Verne V. Smith ${ }^{4}$ \\ ${ }^{1}$ Departamento de Fisica, Universidad de Concepción, Casilla 160-C, Concepción, Chile \\ email: dgeisler@astro-udec.cl \\ ${ }^{2}$ Department of Astronomy, University of Florida, 211 Bryant Space Science Center, \\ Gainesville, Florida, USA 32611-2055 \\ email: aaron@astro.ufl.edu, ata@astro.ufl.edu \\ ${ }^{3}$ University of Minnesota, Department of Astronomy, 116 Church St. SE, Minneapolis, \\ Minnesota USA 55455 \\ email: cole@astro.umn.edu \\ ${ }^{4}$ US Gemini Project Office, National Optical Astronomy Observatories, PO Box 26732, \\ Tucson, Arizona USA 85726-6732 \\ email: vsmith@noao.edu
}

\begin{abstract}
We present results from a project aimed at better understanding the kinematics and metallicities of populous clusters in the LMC. In an effort to update previous $[\mathrm{Fe} / \mathrm{H}]$ determinations, we have utilized FORS2 on the VLT to obtain infrared spectra for more than 200 stars in $28 \mathrm{LMC}$ clusters. The absorption lines of the calcium II triplet were then used to calculate radial velocities and $[\mathrm{Fe} / \mathrm{H}]$ for a sample of clusters spanning a large range of ages $(\sim 1-13 \mathrm{Gyr})$ and metallicities $(-0.3>[\mathrm{Fe} / \mathrm{H}]>-2.0)$. We determine mean cluster velocities to typically $1.6 \mathrm{~km} \mathrm{~s}^{-1}$ and mean metallicities to $0.04 \mathrm{dex}$ (random error). For eight of these clusters, we report the first spectroscopically determined metallicities based on individual cluster stars, and six of these eight have no published radial velocity measurements. Combining our data with archival HST/WFPC2 photometry, we find the newly measured cluster, NGC 1718, is one of the most metal-poor $([\mathrm{Fe} / \mathrm{H}] \sim-0.80)$, intermediate age $(\sim 2 \mathrm{Gyr})$ inner disk clusters in the LMC. Similar to what was found by previous authors, this cluster sample has motions consistent with that of a single rotating disk system, with no indication that the newly reported clusters exhibit halo kinematics. Additionally, our findings confirm previous results which show that the LMC lacks the metallicity gradient typically seen in non-barred spiral galaxies, suggesting that the bar is driving the mixing of stellar populations in the LMC. However, in contrast to previous work, we find that the higher metallicity clusters $(\geqslant-1.0 \mathrm{dex})$ in our sample show a very tight distribution (mean $[\mathrm{Fe} / \mathrm{H}]=-0.48, \sigma=0.09$ ), with no tail toward solar metallicities. The cluster distribution is similar to what has been found for red giant stars in the bar, which indicates that the bar and the intermediate age clusters have similar star formation histories. This is in good agreement with recent theoretical models that suggest the bar and intermediate age clusters formed as a result of a close encounter with the SMC $\sim 4$ Gyr ago.
\end{abstract}

Keywords. galaxies: star clusters, Magellanic Clouds

\section{Acknowledgements}

D.G. gratefully acknowledges support from the Chilean Centro de Astrofísica FONDAP No. 15010003. AJG was supported by NSF CAREER grant AST-0094048 to AS. AAC was supported by a fellowship from the Netherlands Research School for Astronomy (NOVA). VVS has been supported by the NSF through grant AST03-07534. 